\title{
Electrical properties of the $\mu$ s pulsed glow discharge in a Grimm-type source: comparison of dc and $\mathrm{rf}$ modes $\dagger$
}

\author{
Varvara Efimova, ${ }^{* a}$ Volker Hoffmann ${ }^{a}$ and Jürgen Eckert ${ }^{a b}$
}

\author{
Received 30th September 2010, Accepted 4th November 2010 \\ DOI: $10.1039 / \mathrm{c0ja00176g}$
}

\begin{abstract}
The electrical properties, in particular the $U-I$ characteristics, current and voltage signal shapes within the pulse, are important parameters for the understanding of the processes taking place in the pulsed glow discharge (PGD). The electrical properties are also closely related to the analytical performance of the PGD such as sputtering rates, crater shapes and emission yields. Moreover, the dependence of the $U-I$ plots on the density of the discharge gas can be used to estimate the gas temperature. This result is relevant for the analysis of thermally fragile samples. Nevertheless, there is a lack of PGD studies where the current and voltage signals are considered in detail. Therefore, this article is dedicated to the electrical properties of PGD. The influence of the PGD parameters (duty cycle and pulse duration) on the electrical properties is examined. The results highlight the optimum parameters for particular analytical applications. The question, whether direct current (dc) and radio frequency (rf) discharges behave similarly is also discussed and all experiments are performed for both modes. The comparative studies reveal strong similarities between dc and rf pulsed discharges.
\end{abstract}

\section{Introduction}

Nowadays pulsed glow discharge (PGD) is becoming evermore popular in mass spectrometry and optical emission spectrometry (OES). The reason is that it has a greater number of attractive features, as compared to continuous discharge sources, which broaden its analytical potential. ${ }^{1,2}$ In PGD the power is not applied continuously but only periodically with a certain frequency. This reduces sample heating during analysis and allows the analysis of thermally fragile samples. ${ }^{3,4}$ In addition, the sputtering rate is reduced which renders PGD preferable for the analysis of thin films. ${ }^{5-8}$ The pulsing process initiates different excitation and ionization mechanisms at the beginning, during and after the pulse with strong, medium and soft plasma conditions. ${ }^{9-11}$ Using this feature and time-of-flight mass spectrometry one can extract elemental and molecular information from gaseous samples. ${ }^{12-15}$ Recent studies have shown the possibility for direct oxidation state determination both from gaseous and solid state materials. ${ }^{16,17}$ By measuring the emission at a defined time of the pulse one can discriminate the impact of contaminations or other species in the plasma. ${ }^{18-20}$

Besides the common parameters of the glow discharge (GD) voltage, current and pressure, in the case of PGD there are additional parameters - duty cycle, pulse duration and pulse frequency. Moreover a wider range of voltages and currents can

${ }^{a}$ IFW Dresden, Institut für Komplexe Materialien, P. O. Box 270116 D-01171 Dresden, Germany. E-mail: v.efimova@ifw-dresden.de; v.hoffmann@ifw-dresden.de; Fax: +49 351 4659 452; Tel: +49 3514659 734 (691)

${ }^{b}$ TU Dresden, Institut für Werkstoffwissenschaft Dresden, D-01062 Dresden, Germany. E-mail: j.eckert@ifw-dresden.de; Fax: +49 3514659 452; Tel: +493514659271

$\uparrow$ This article is part of a themed issue highlighting the latest work in the area of Glow Discharge Spectroscopy, including work presented at the International Glow Discharge Spectroscopy Symposium 2010, August 22-25, Albi, France. be applied. From one side, as mentioned before, more discharge parameters enable one to control the heating and sputtering rate of the sample with greater precision. On the other side it complicates the analysis. Thus, understanding the influence of all the parameters on the discharge performance is important. One of the keys to understand the glow discharge is its electrical behaviour. The electrical properties are also closely related to the analytical performance of PGD. For example, from the voltagecurrent $(U-I)$ characteristics one can see if the discharge is operating in the abnormal mode, which is extremely important for homogeneous sample sputtering and efficient excitation and ionization. ${ }^{21,22}$ It has also been shown that the slope of the $U-I$ characteristics changes with the temperature of the discharge system. ${ }^{23,24}$ This effect stimulated the idea to estimate the discharge gas temperature from the $U-I$ characteristics of PGD. ${ }^{25}$

Measurement of $U-I$ characteristics in PGD is complicated by the fact that their behaviour changes within the pulse. ${ }^{26}$ Also the shape of the crater ${ }^{7}$ and the emission intensity at any characteristic wavelength are directly connected to the voltage and current signals during the pulse. ${ }^{27}$ Although the influence of the pulse parameters on the crater shape $\mathrm{e}^{7}$ and emission intensity ${ }^{28-30}$ is considered, less attention has been paid to the electrical properties of the PGD.

Summarizing the above-mentioned facts, the electrical properties, i.e. the $U-I$ characteristics as well as current and voltage signal shapes, are important in understanding PGD processes. They are also necessary for plasma modelling studies. $^{31,32}$ Nevertheless, there is a lack of such studies.

Therefore, in the current work the influence of the PGD parameters (pulse duration, pulse frequency and duty cycle) on the $U-I$ characteristics, and current and voltage signal shapes is investigated. Pulsed direct current (dc) and radio frequency (rf) discharges are compared. In the future correlation of these electrical parameters with crater shapes and light emission will be considered. 


\section{Experimental}

\subsection{Experimental setup}

For dc and pulsed dc measurements a $4 \mathrm{~mm}$ Grimm-type source from Spectruma (GDA-750, Spectruma) was used. The discharge pressure was measured in the anode body by a Pirani gauge, calibrated by a Baratron manometer (MKS Baratron Type 127; range $0-100 \mathrm{hPa}$ ). A high voltage generator (MCN 350-2000, FUG) was used to create continuous dc GD. The pulsed dc GD was excited with a high voltage pulse generator (RUP 3-3a, GBSElektronik $\mathrm{GmbH}$ ) and synchronized by an external TTL pulse generator (33120A, HP). The discharge voltage and the current of the pulsed discharge were transferred via a digital oscilloscope (11201, Tektronix) to the computer for further evaluation.

For rf and pulsed rf measurements a modified Grimm-type glow discharge source with a $4 \mathrm{~mm}$ anode tube was used. The glow discharge was excited with a free running of generator (Forschungstechnik IFW, $3.37 \mathrm{MHz}$ ). The measurements of transient voltage and current were made by voltage and current probes integrated into the source. The experimental setup and the measurement system are described in detail in the paper of Wilken et al. ${ }^{33}$ Additionally, the $\mathrm{rf}$ voltage was measured by a $1: 100$ voltage divider (GE 3425, General Elektronik GmbH)). The signals from the voltage and current probes were fed into a high speed oscilloscope (LC584A, LeCroy) from which they were subsequently transferred into a PC, where further evaluation was conducted.

For all electrical experiments $10 \mathrm{~mm}$ thick pure $\mathrm{Cu}$ samples $(\varnothing$ $30 \mathrm{~mm}$ ) were used.

\subsection{Optimization of the experimental setup}

Before performing any experiments it is important to ensure that the measured signals correspond to the real ones and to reveal possible measurement artefacts.

In the case of a pulsed dc system it has been shown that the electronic circuit of the pulsed power supply influences the current and voltage signal shapes. ${ }^{25,34,35}$ For example, with one pulse generator the voltage and current signals disappear abruptly after pulse termination, but with another, both signals decrease gradually ${ }^{25}$ Hence, the origin of the voltage and current signals after pulse termination is not in the plasma, which must be taken into account for GD process studies. To avoid this measurement artefact, the power supply, RUP 3-3a, used in the current work produced a rectangular voltage signal.

The power supply for rf discharge can be of two types: a fixed frequency generator with matching box or a free running generator. The first type, the most often used, works at an exact or defined frequency. So as to transfer the maximum power from the generator to the discharge the output impedance of the generator should be matched to the input impedance of the discharge source with the help of a matching box. The matching process can take up to several seconds, which determines the plasma stabilisation time. For a free running generator no matching box is needed because the source is a part of the oscillating circuit. ${ }^{36,37}$ This simplifies the generator operation and reduces the stabilisation time. The oscillation frequency changes slightly and thus compensates the variation of the plasma impedance. Despite differences in the electronic circuits, both generator types create the same plasma conditions. Therefore, it does not matter which type is used. In this work a free running generator was used.

For analytical applications, the GD source is capacitively coupled to the $\mathrm{rf}$ generator. This means that the rf voltage is delivered to the source through a capacitor. In the case of conductive samples the capacitor is responsible for biasing the rf voltage to a negative value. This causes the bombardment of the sample by positive ions and its sputtering. ${ }^{37}$

In case of pulsed $\mathrm{rf}$ discharges, some limitations to the capacitance value of the capacitor exist. Fig. 1 shows the influence of the capacitance on the bias voltage behaviour.

The lower the capacitance value, the higher the voltage loss at the capacitor. In Fig. 1(a) the low rf voltage, caused by the voltage loss at the capacitor, is not sufficient to ignite the plasma. Thus, the rf voltage oscillates around the zero value and no sample sputtering occurs.

If the capacitance is too large it decelerates or prevents the decrease of the bias voltage after the pulse. In this case an overlay of $\mathrm{rf}$ (during the pulse) and dc (between the pulses) voltages is obtained (Fig. 1(b)). Only with an optimal capacitance value is a normal pulsed rf voltage signal provided (Fig. 1(c)). The optimal capacitance value depends on the discharge system and should be established for each system separately.

\section{Results and discussion}

\subsection{Pulsed direct current and radio frequency discharges. Is there any difference?}

PGD can be operated either in dc or in $\mathrm{rf}$ mode. The main advantage of the rf mode is its ability to sputter nonconductive samples. Sometimes the rf mode is also advantageous for the analysis of conductive samples, because it is less affected by nonconductive surface oxide contamination. ${ }^{38}$ But it is questionable whether this is the only difference between the rf and dc modes. In the literature one can find quite different information when comparing $\mathrm{dc}$ and $\mathrm{rf}$ discharges. In the following text references will be highlighted where some authors suppose that ionisation and sputtering are different in dc and $\mathrm{rf}$ modes, and others who believe that both modes are similar.

It has been reported that in $\mathrm{rf}$ discharges two different discharge regimes exist: $\alpha$ and $\gamma^{2,39,40}$ In the $\alpha$ regime the electrons in the bulk plasma are heated by the rf field oscillations. These electrons have a high enough energy to cause ionisation and sustain the plasma. In the case of the $\gamma$ regime the discharge is sustained by the electrons emitted from the cathode (secondary electrons). In dc discharges only the $\gamma$ regime exists. The absence of the $\alpha$ regime and also computation results ${ }^{39}$ indicate that the ionization in dc mode is less efficient than in the rf one. However, later modelling studies have shown that the calculated $\mathrm{Cu}$ atom and ion densities, optical emission spectra, sputtering rates and sputter crater shapes are similar for $\mathrm{rf}$ and dc mode. These computational results are in good agreement with experimental data.$^{41}$ Lewis et al. studied the temporal emission characteristics of pulsed dc and rf discharges and found only negligible differences between dc and $\mathrm{rf}$ mode. ${ }^{34}$ Präßler et al. observed that the GD OES intensity-time profiles, crater shapes and depth resolution are similar for $\mathrm{rf}$ and de discharges. ${ }^{42}$ On the other hand, 


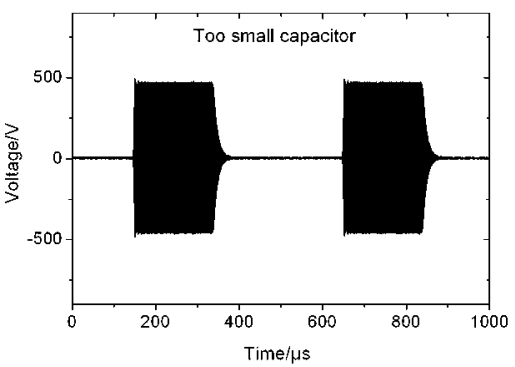

(a)

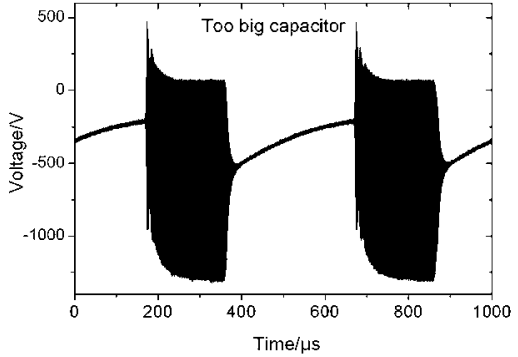

(b)

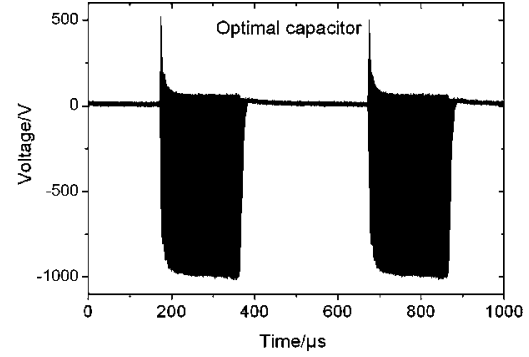

(c)

Fig. 1 The pulsed rf voltage measured with application of (a) small $22 \mathrm{pF}$ (b) big $2000 \mathrm{pF}$ and (c) optimal capacitor $95 \mathrm{pF}$ between the rf generator and the source (effective voltage $700 \mathrm{~V}$, pressure $6 \mathrm{hPa}$, duty cycle 0.4 , pulse duration $200 \mu$ s, frequency $2 \mathrm{kHz}$ ).

Harrison mentioned that the efficiency of sputtering by an $\mathrm{rf}$ discharge is somewhat less than that obtained by dc. ${ }^{38}$

Thus, from the literature it is difficult to conclude either that the dc and rf modes are different or similar. At this point it is extremely important to emphasize which electrical conditions between modes are compared. Two discharges can be compared at the same pressure and only in the case when the power they are consuming is the same. For the case of rf discharges an equivalent to the dc-voltage and dc-current is necessary. Bogaerts et al. noticed that in the dc mode a higher voltage than the rf bias value is required to yield the same power value as in the rf-mode. ${ }^{39}$ Additional studies ${ }^{43}$ and subsequent work ${ }^{37}$ have shown that the rf equivalent of the dc voltage is the effective voltage $U_{\text {eff }}$, which is the cycle root mean square value of the periodic voltage signal $U_{\mathrm{p}}(t)$ plus bias $U_{\text {bias }}$ (see eqn (1)). The equivalent for the current value $I_{\text {eff }}$ is the rf power $P$ divided by the effective voltage (see eqn (2)-(4)).

$$
\begin{gathered}
U_{\text {eff }}=\sqrt{\frac{1}{t_{\mathrm{p}}} \int_{\mathrm{o}}^{t_{\mathrm{p}}}\left(-U_{\text {bias }}-U_{\mathrm{p}}(t)\right)^{2} \mathrm{~d} t} \\
P(t)=U_{\mathrm{p}}(t) I_{\mathrm{p}}(t) \\
P=\frac{1}{t_{\mathrm{p}}} \int_{0}^{t_{\mathrm{p}}} P_{\mathrm{t}}(t) \mathrm{d} t \\
I_{\text {eff }}=\frac{P}{U_{\text {eff }}}
\end{gathered}
$$

With these parameters the best agreement of the sputtering rates and emission yields between $\mathrm{rf}$ and dc discharges was obtained. For example, the power-voltage dependences are similar for $\mathrm{rf}$ and dc modes if the effective voltage is used in the $\mathrm{rf}$ case. ${ }^{26}$ Apparently, the dc and $\mathrm{rf}$ discharges are very similar, if the correct electrical conditions are chosen.

It must be mentioned that the determination of power, $\mathrm{rf}$ voltage and dc bias deteriorates with increasing sample thickness. The quality of the power measurement at fixed frequency generator systems depends on the power losses in the matchbox and the quality of the used correction - the so-called subtraction method. The rf voltage becomes lower throughout nonconductive samples, depending on sample thickness, frequency and current. In principle, this voltage loss can be corrected, if voltage and current are measured. The direct measurement of the $\mathrm{dc}$ bias becomes totally impossible and calculation procedures ${ }^{37}$ or estimations ${ }^{43}$ must be used.

As was shown in the Introduction, the study of electrical parameters is important and can also help to answer questions about the comparative features of $\mathrm{dc}$ and $\mathrm{rf}$ discharges. In the next sections the electrical parameters of pulsed $\mathrm{rf}$ and $\mathrm{dc}$ discharges are studied and compared. The effective voltage is used in the case of an rf discharge so as to obtain the same plasma conditions as in dc mode.

\subsection{Voltage-current characteristics of the pulsed glow discharge}

For all analytical applications of the Grimm-type source, the GD is operated in the abnormal mode, where flat craters and high power are obtained. In this mode the discharge conditions are selected such that the current flows homogeneously through the cathode surface (for $4 \mathrm{~mm}$ source: voltage 500-1500 V, current 10-100 $\mathrm{mA}$, pressure $2-15 \mathrm{hPa}$ ). The expansion of the plasma over the cathode is obstructed by the anode. Therefore, at higher voltages more electrons are extracted from the surface per unit area and the current increases. This process defines the form of the voltage-current characteristics of the GD: the current increases with voltage (Fig. 2(a)). One can also see the saturation of the current at higher voltages and pressures in a dc discharge. This effect is well known in the case of continuous de discharge and is explained by the heating of the discharge gas when high power is applied. ${ }^{23,24,26}$

The voltage-current characteristics measured for $\mathrm{rf}$ discharges within the last $600 \mathrm{~ns}$ of the pulse show a linear behaviour without saturation in the ionic part (see Fig. 2(b)). This indicates that during the short time of some rf periods the gas temperature stays constant.

For the pulsed dc and rf discharges, the influence of the duty cycle on the voltage-current characteristics was studied.

In the case of pulsed dc discharge it was essential to choose a convenient type of current signal acquisition. There are two possibilities to record the current signal with the high voltage pulse generator RUP 3-3a. One of them provides a high time resolved measurement of the current within the pulse. The other gives the total input current values in the pulser. So, for the voltage-current plots one can either measure the current within the pulse and calculate the average value during the pulse 


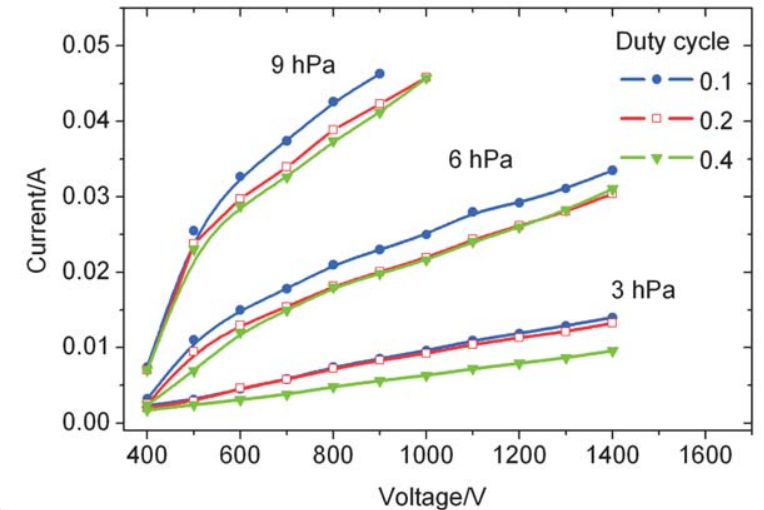

(a)

Fig. 2 Voltage-current plots of pulsed (a) dc (presenting the averaged current values within the $200 \mu$ s pulse) and (b) rf (measured in the last $600 \mathrm{~ns}$ of the $50 \mu$ s pulse) discharges.

on-time (way 1) or measure the input current and divide it by the duty cycle (way 2). Both ways produce an averaged current value during one pulse. Experiments have shown that the current measured by way 2 exceeds the current measured by way 1 by $15 \%$ (see Fig. 3). A part of this difference can be explained by the consumption of power by the pulse generator, which is converted to heat. Therefore, way 1 is preferable, if accurate currents are needed. However, as the ratio between both currents is stable and does not depend on the voltage, it is possible to correct the current measured by way 2 . Because way 2 is much easier to perform and even possible on a commercial scale, this later current recording way (2) was used in the present work.

The experimental data (Fig. 2(a)) show that at a constant pulse duration $(200 \mu \mathrm{s})$ the average current during the pulse on-time changes with the duty cycle. At $3 \mathrm{hPa}$ the plot, which corresponds to the 0.4 duty cycle, is placed lower than the plots for the 0.2 and 0.1 duty cycles. At 6 and $9 \mathrm{hPa}$ the decrease of the current is already seen for the 0.2 and 0.4 duty cycles.

The measurement system developed at the IFW $^{33}$ allows recording of the rf current and voltage during the pulse, but because of the capacitive coupling the average current becomes zero. Therefore, in Fig. 2(b) the measured voltage-current plots

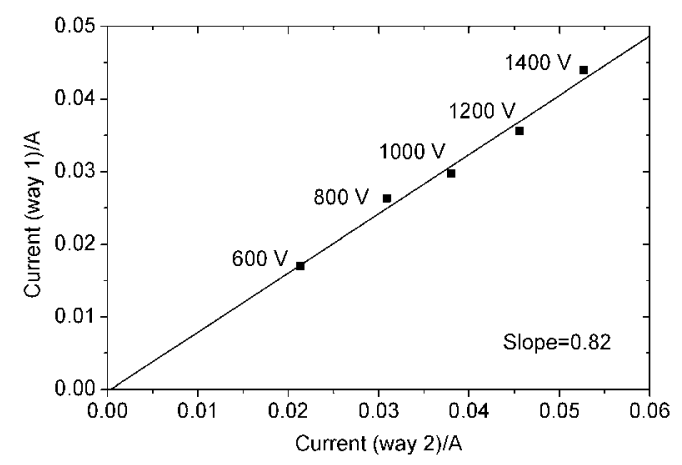

Fig. 3 The current values within one pulse acquired at different voltages by measurement of the total input current of the pulser and division by the duty cycle ( $x$-axis) and by the measurement of the current within the pulse and calculation of the average value during the pulse ( $y$-axis) (pressure $6 \mathrm{hPa}$, duty cycle 0.2 , pulse duration $100 \mu \mathrm{s}$, frequency $2 \mathrm{kHz}$ ).

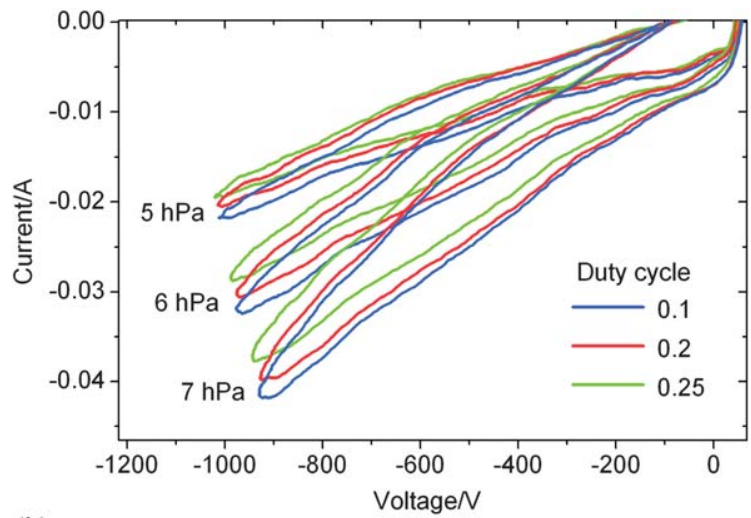

(b)

correspond to a few oscillation periods of the $\mathrm{rf}$ current and voltage at the end of the $50 \mu$ s pulse. One can see only the ionic part of the voltage-current characteristics, where the voltage is negative. This part is responsible for cathode sputtering and takes most of the rf cycle time. As in the dc case, a decrease of the current at higher duty cycles can be seen (Fig. 2(b)). In the positive region no changes were observed at different duty cycles.

This raises the question, why the voltage-current graphs for constant pulse length but different duty cycles are not congruous? Hoffmann et al. ${ }^{26}$ have already mentioned the decrease of the current with duty cycle and explained it by the heating of the discharge gas at high duty cycles. It was also observed that plots measured at the beginning and at the end of the same pulse are different. Moreover, this difference depends on the pulse parameters. On the other hand, it requires a lot of work and time to measure the voltage-current characteristics at different times of the pulse, varying duty cycle, frequency, pulse duration and try to explain the reason of these effects. Therefore, for the further study and understanding of the PGD behaviour it is important to look at the current and voltage signal shapes during the pulse.

\subsection{Voltage and current signal shapes within the pulse}

As already mentioned in the experimental part (Section 2.2), the dc pulse generator used in this work produces a rectangular voltage signal. Therefore, this part will focus on the current shapes during the pulse. Nevertheless there is one aspect which should be mentioned here. Contrary to the dc mode, the voltage signal produced by the $\mathrm{rf}$ generator is not rectangular at the beginning of the pulse (see Fig. 4).

This effect has already been described by Wilken et al. ${ }^{37}$ At the beginning of the pulse the electrons remain at the cathode surface and charge it up to a negative bias voltage. This procedure takes around $50 \mu \mathrm{s}$ and depends on the capacitance of the system. The described effect is not a measurement artefact and should be taken into account when pulsed $\mathrm{dc}$ and $\mathrm{rf}$ discharges are compared. It is also important to know that when very short pulses are chosen (shorter than $50 \mu \mathrm{s}$ ), the voltage will not reach its prescribed value (see Fig. 4). This will lead to a decrease of the sputtering rate. 


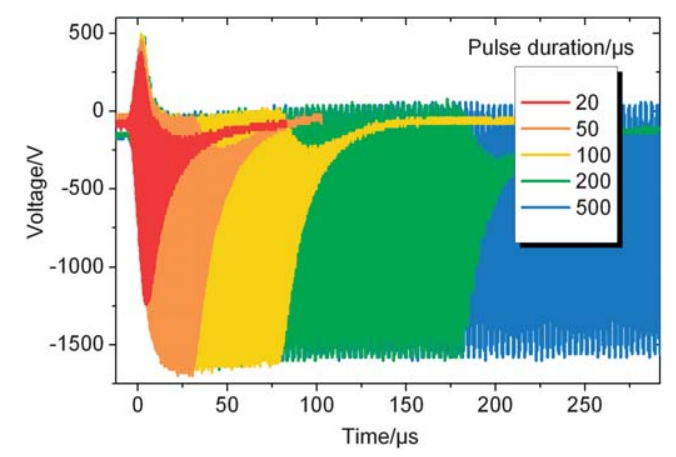

Fig. 4 Rf voltage signal measured at different pulse durations (effective voltage $800 \mathrm{~V}$, pressure $6 \mathrm{hPa}$, duty cycle 0.2 ).

The influence of the discharge parameters on the current signal shapes during the pulse was studied and is described below. The following discharge parameters are considered: voltage, duty cycle, pulse duration and frequency.

3.3.1. Influence of the voltage on the current shapes. For the presentation of voltage-current characteristics of the pulsed dc discharge, the average current value over the pulse was evaluated (see Fig. 2(a)). Such averaging hides possible effects during the pulse. Therefore, now the current shapes during the pulse are presented at different voltages (see Fig. 5). The other discharge parameters such as pressure, duty cycle, frequency and pulse duration were fixed.

As expected, the current within the pulse shown in Fig. 5 increases with increasing voltage. But there is one additional effect, which was not visible before: the current peak at the beginning of the pulse becomes more pronounced at higher voltages. The high current peak at the beginning of the pulse has already been observed by others. ${ }^{20,35,44}$ Because of the strong excitation and ionization conditions, the region at the beginning of the pulse is often used in time-gated measurements to extract elemental information from the sample. ${ }^{14}$ However, the origin of the high current after the plasma ignition is still not clear. In the modelling studies, in order to predict the high current peak at the beginning of the pulse, a considerable rise of the gas temperature in this region was assumed. ${ }^{31}$ Later theoretical studies assumed that the initial current peak is attributed to the measuring circuit and not to the real plasma current. ${ }^{45}$ There is a theory that a part of this high current is the capacitive current caused by charging of a capacitor (the discharge source without the plasma). ${ }^{2}$ The

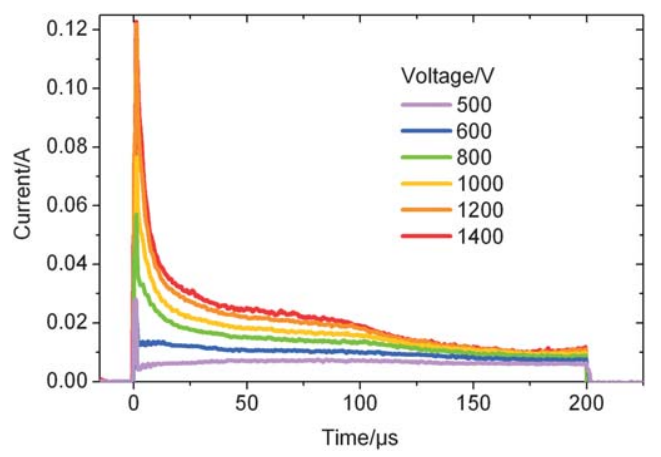

Fig. 5 De current signal within the pulse measured at different voltages (pressure $6 \mathrm{hPa}$, duty cycle 0.2 , pulse duration $200 \mu \mathrm{s}$, frequency $1 \mathrm{kHz}$ ). discharge source used in this work, including cables, has a capacitance $C$ around $230 \mathrm{pF}$. The resistance value $R$ which is between the power supply and the source is $35 \mathrm{Ohm}$. In this case, the application of the voltage $U_{0}$ across the $230 \mathrm{pF}$ capacitor will produce a huge current peak $I_{\max }$ of several amperes, but it will decay with time $t$ in some ns (see eqn (5)-(6)).

$$
\begin{array}{r}
I=I_{\max } \mathrm{e}^{-t / R C} \\
I_{\max }=\frac{U_{0}}{R}
\end{array}
$$

The huge (several A) peak in the first ns of the pulse probably exists, but it is not possible to record it with our equipment, because the probes are not designed for such high frequencies and currents. Hence this does not explain the commonly observed current peak in the $\mu$ s region, as depicted in Fig. 5. In addition, the capacitive current should depend linearly on the applied voltage. This is in contradiction with Fig. 5 where the current peak disappears at low voltages. Therefore, further studies on this topic are necessary.

To check if the pulsed rf discharge reveals a similar effect, the rf current signal shapes were measured at different effective voltages and all other discharge parameters were kept constant. In Fig. 6(a) rf current and voltage signals measured with Wilken's ${ }^{37}$ system at the end of the $200 \mu$ s pulse are presented. By compressing the time scale one can see the whole picture of the rf current during the pulse. It is interesting that the behaviour of the pulsed rf discharge is similar to the dc case (see Fig. 6(b)) and current peaks appear in the prepeak region as well as in the electron (positive) and ionic (negative) current part.

In comparison with the plateau region the current peaks at the beginning of the rf pulse are not as high as in the dc case. But one can clearly see the similarity between rf and dc pulses: the peaks disappear at lower voltages.

3.3.2. Influence of the duty cycle, pulse duration and frequency on the current shapes. The main difficulty in studying the influence of the duty cycle, pulse duration and frequency on the current shapes is that these discharge parameters cannot be varied separately. This situation is similar to that with voltage, current and pressure of the glow discharge. One cannot change for example the voltage without changing current or pressure. Because it was found that the contribution of the pressure into the glow discharge performance is small, it was implicitly included in the current and potential functions. ${ }^{46}$ Also Boumans equation for sputtering does not include pressure, and sputtering can be simply described by a linear dependence on voltage and current. ${ }^{47}$ However, for a defined material it is possible to calculate the voltage as a function of current and pressure or the current as a function of voltage and pressure, but the resulting equations will be more difficult.

Similarly as for the pressure in this work the frequency was found to be a less important parameter. The reason is that (1) the duty cycle is directly correlated with the power introduced into the discharge and (2) the pulse duration is quite important for the study of the current shape during the pulse. Hence, duty cycle and pulse duration were independently varied while the frequency was allowed to reach its own value depending on the first two parameters. 

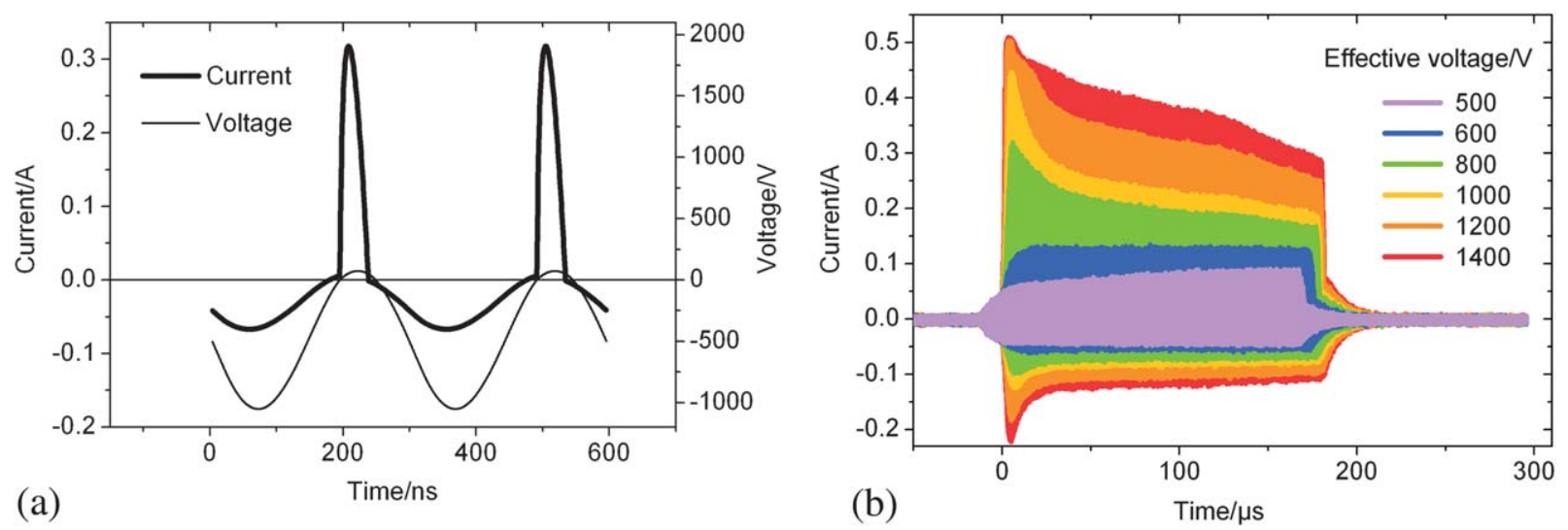

Fig. 6 (a) Current and voltage signals of the rf discharge (measured in the last $600 \mathrm{~ns}$ of the $200 \mu$ s pulse, effective voltage $1400 \mathrm{~V}$, pressure $6 \mathrm{hPa}$, duty cycle 0.2 , frequency $1 \mathrm{kHz}$ ) and (b) rf current signal within the pulse measured at different effective voltages (pressure $6 \mathrm{hPa}$, duty cycle 0.2 , pulse duration $200 \mu \mathrm{s}$, frequency $1 \mathrm{kHz}$ ).
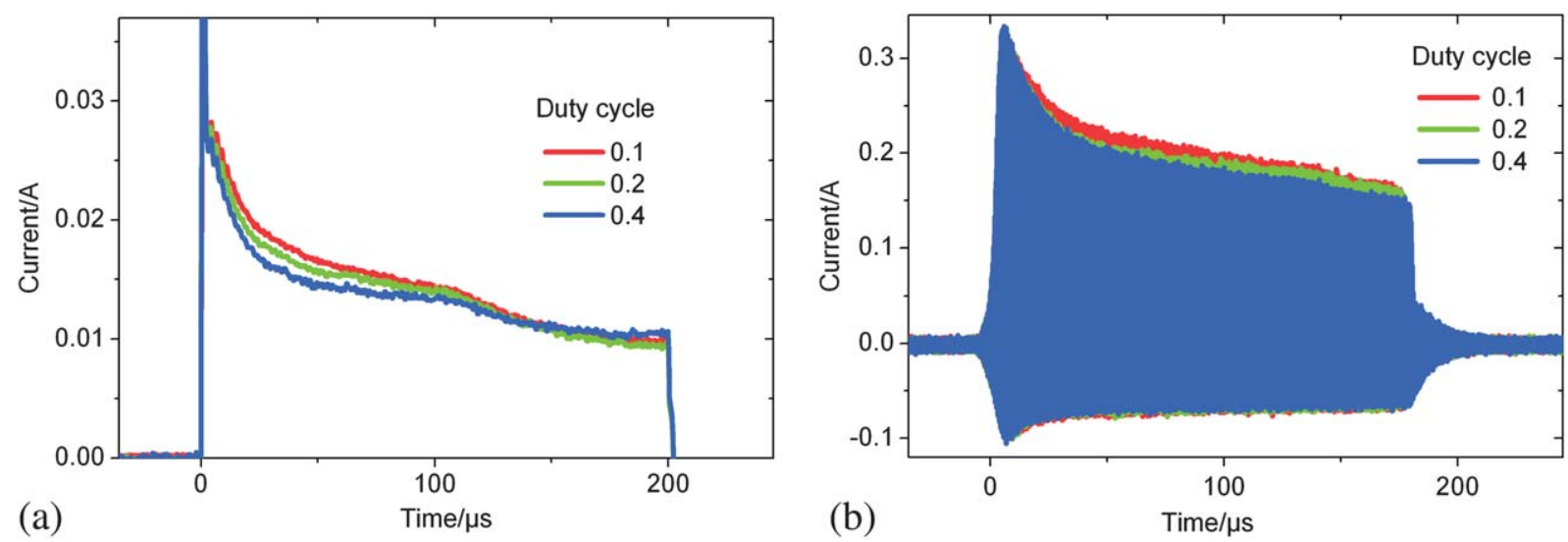

Fig. 7 Dc (a) and rf (b) current signals within the pulse measured at different duty cycles ( $6 \mathrm{hPa}, 800 \mathrm{~V}$ (in the case of rf $\left.U_{\text {eff }} 800 \mathrm{~V}\right)$, pulse duration $200 \mu \mathrm{s})$.

In this way the influence of the duty cycle on the current was studied with a fixed pulse duration. Fig. 7 presents dc and rf current signals with $200 \mu$ s pulse length at different duty cycles. The shape of the current signal remains unchanged for different duty cycles. But one can see that the current level at higher duty

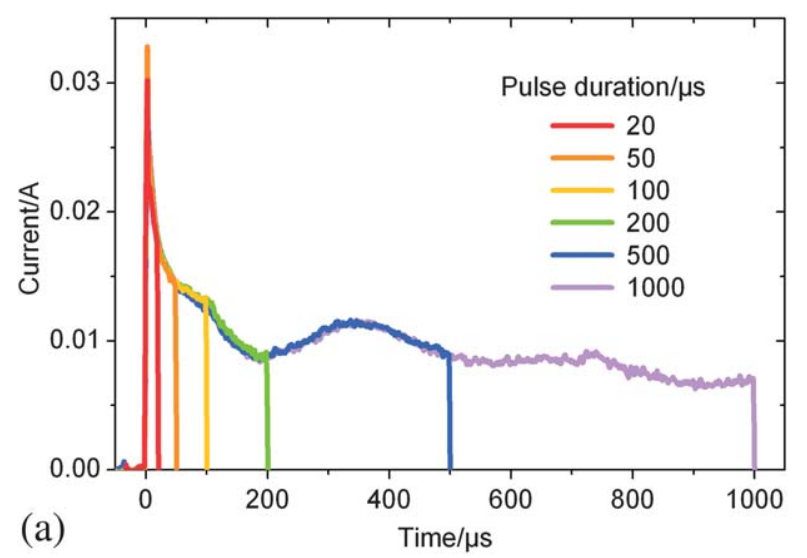

cycles is lower for both de and $\mathrm{rf}$ modes. This is the reason why the voltage-current characteristics measured with different duty cycles are not congruous in spite of the correction to the duty cycle (see Section 3.2 Fig. 2). As already discussed above, the decrease of the current is caused by the discharge gas heating

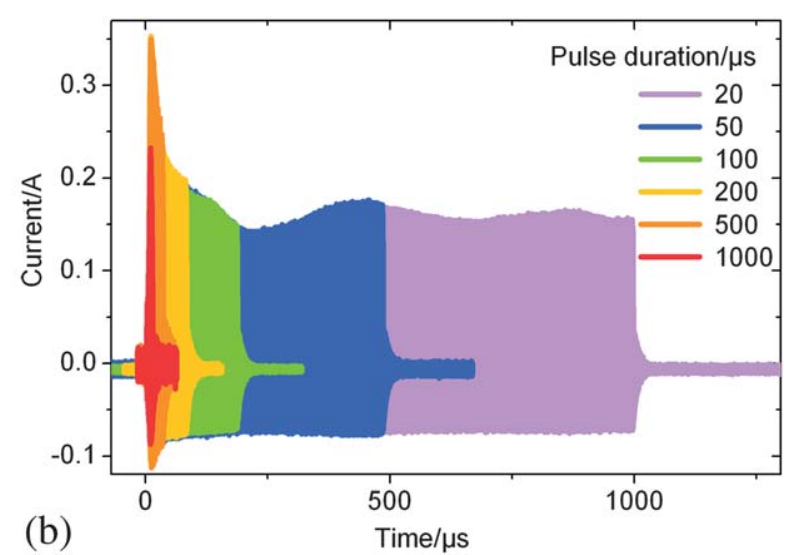

Fig. 8 Dc (a) and rf (b) current signals within the pulse measured at different pulse durations (6 hPa, $800 \mathrm{~V}$ (in the case of rf effective voltage $800 \mathrm{~V})$, duty cycle 0.2 ). 


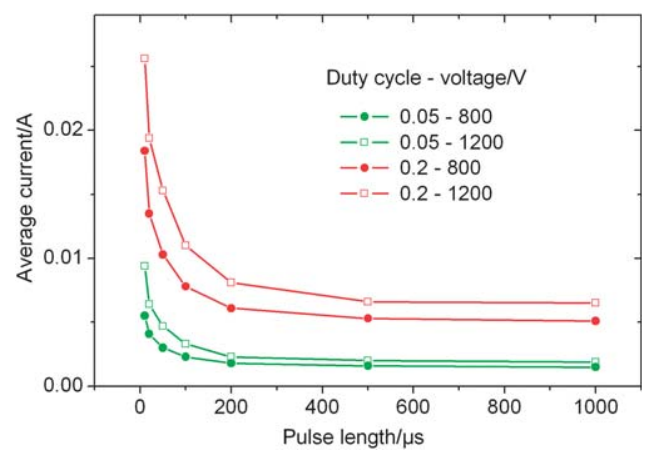

Fig. 9 Dependence of the pulser input current (not divided to the duty cycle) on pulse length $(6 \mathrm{hPa})$ in pulsed dc.

when higher power is applied. The power can be increased either by an increase of the voltage and current, or by an increase of the duty cycle. In the first case one observes a curvature of the voltage-current plots (Fig. 2a) and in the second case the decrease of the current within the pulse despite voltage and pressure is fixed (Fig. 7).

Since the effect of the duty cycle is understood, the influence of the pulse duration on the current at fixed duty cycle was studied. In Fig. 8 the $\mathrm{dc}$ and $\mathrm{rf}$ currents measured at different pulse durations but with a fixed duty cycle, voltage (in the case of $\mathrm{rf}$ discharge - effective voltage) and pressure are presented. First of all it should be emphasized that the current for all pulse durations follows the same trajectory and does not drop as in the case of higher duty cycles in Fig. 7.

Nevertheless, the current signal changes with time and long pulses look completely different than short pulses. In general one can see a decrease of the current with the time of the pulse. Therefore, in spite of the same duty cycle, longer pulses mean lower average current and very probably also a lower sputtering rate and emission intensity. Fig. 9 demonstrates the dependence of the total input current on pulse duration with a fixed duty cycle. The reduction of this current from the shortest to the longest pulse is around $75 \%$. This effect can also be explained by heating of the discharge gas when the discharge runs for a longer period. However, if this is the case, then why does the current not decrease gradually within the pulse?

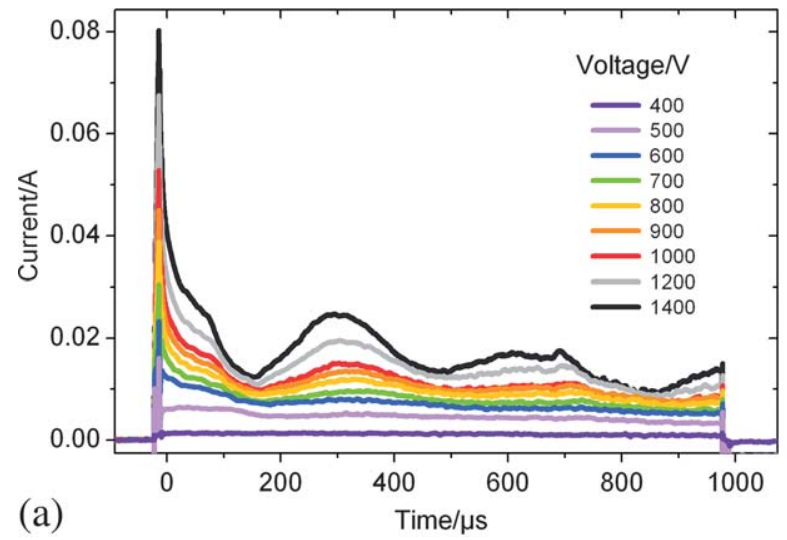

In Fig. 8 one can also see reproducible oscillations of the current signal. Moreover, the oscillations appear for both dc and rf pulses. A very similar phenomenon was previously observed by Nelis et al. ${ }^{27}$ Periodical variations of the $\mathrm{Cu} 224.7 \mathrm{~nm}$ light emission with a period of $200 \mu$ s were observed, which disappeared after $1 \mathrm{~ms}$. Nelis suggested that the observed oscillations were caused by a variation of the discharge gas particle density due to changes in either pressure or temperature. This explanation can also be valid for the current oscillations observed in this work, but further studies are necessary. The main question is why does the pressure or temperature oscillate after discharge ignition? Possible mechanisms responsible for the generation of pressure waves are discussed in the paper of Voronov et al. $^{48}$

It is found that the oscillations disappear at low voltages, similarly as the peak at the beginning of the pulse (Fig. 10).

\section{Conclusions}

This work covers the electrical features of PGD, which are crucial for both understanding the discharge processes and analytical applications.

Firstly, the importance of the optimization of the measurement of voltage and current signals within the pulse was shown. Possible artefacts which are not caused by the discharge processes, but by the electrical circuit of the pulsed system were described.

The difficulties in comparing dc and rf modes were also discussed. For these comparative studies in this work the effective voltage in the rf mode was used as an equivalent to the dc voltage. Nearly all phenomena found with de discharges also appear in the rf case. No significant difference between rf and dc pulsed discharges was observed. For both, rf and dc discharges, a decrease of the current caused by heating of the discharge gas at higher powers was found. In addition, the current signal shape within the pulse showed a similar behaviour in the rf and dc modes: (1) growth of the current peak at the beginning of the pulse at higher voltages, (2) a drop of the current level within the pulse when a higher duty cycle is applied and (3) oscillations of the current signal within long (up to $1 \mathrm{~ms}$ ) pulses. Thus, in terms of the electrical properties, the pulsed rf and dc discharges are very similar.

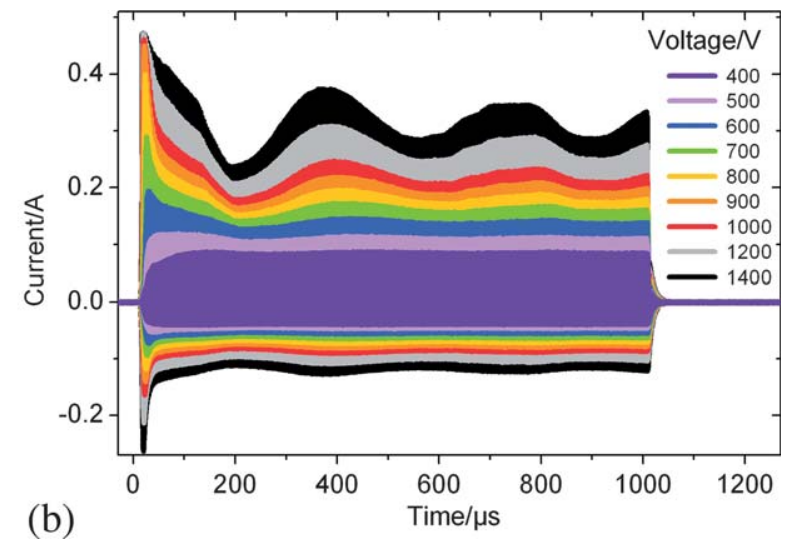

Fig. 10 Dc (a) and rf (b) current signals within the pulse measured at different voltages (in the case of rf effective voltages) (pressure 6 hPa, duty cycle 0.2 , pulse duration $1000 \mu \mathrm{s}$, frequency $0.2 \mathrm{kHz}$ ). 
The influence of the PGD parameters (pulse duration, pulse frequency and duty cycle) on the $U-I$ characteristics and current and voltage signal shapes was also investigated. The voltagecurrent plots indicate heating of the discharge gas when operating at high duty cycles. This result is quite important for the analysis of thermally fragile samples. Nevertheless, more in depth studies on the behaviour of the signals within one pulse are necessary.

The measured voltage signal shape is rectangular and is not influenced by the pulse parameters. One should only take into account that the rf voltage needs around $50 \mu$ s to reach its prescribed value because of the bias voltage formation. Hence, the use of very short pulses can lead to a decrease of the sputtering rate.

Opposite to the voltage, the current signal is not rectangular within the pulse. There is a current peak in the first $\mu$ s of the pulse. Afterwards the current decreases and oscillations with a period of around $200 \mu$ s occur. As the current signal is not constant during the pulse, the pulse duration is an important parameter. The experimental results show that with a fixed duty cycle the average discharge current depends on the pulse duration. The shorter the pulse the higher the average current. Presumably, the sputtering rate will also be higher with short pulses, in spite of the fixed duty cycle.

Very probably the emission intensity within the pulse will follow the current signal shape. A corresponding investigation at different duty cycles and pulse durations is currently in progress.

In this work the nature of the oscillations of the current signal within the pulse and the high current peak at the beginning of the pulse are not clarified. Therefore, further studies of these phenomena are necessary. Moreover, a detailed investigation of the sputtering rates and the emission intensities of the PGD considering the results on the electrical properties should follow.

\section{Acknowledgements}

The authors kindly acknowledge the financial support from the FP6 Research Training Network GLADNET (No. MRTN-CT2006-035459) and collaboration with Michael Analytis (Spectruma Analytik $\mathrm{GmbH})$.

\section{Notes and references}

1 W. W. Harrison, J. Anal. At. Spectrom., 1998, 13, 1051.

2 Ph. Belenguer, M. Ganciu, Ph. Guillot and T. Nelis, Spectrochim. Acta, Part B, 2009, 64, 623.

3 C. Pan and F. L. King, Appl. Spectrosc., 1993, 12, 2096.

4 D. Pollmann, K. Ingeneri and W. W. Harrison, J. Anal. At. Spectrom., 1996, 9, 849.

5 C. Yang, K. Ingeneri, M. L. Mohill and W. W. Harrison, J. Anal. At. Spectrom., 2000, 15, 73.

6 K. Wagatsuma, ISIJ Int., 2004, 44, 108.

7 E. Oxley, C. Yang and W. W. Harrison, J. Anal. At. Spectrom., 2000, 9, 1241 .

8 C. Yang, K. Ingeneri, M. L. Mohill and W. W. Harrison, Anal. Chem., 1999, 71, 5328.

9 G. P. Jackson and F. L. King, Spectrochim. Acta, Part B, 2003, 58, 1417.

10 G. P. Jackson, C. L. Lewis, S. K. Doorn, V. Majidi and F. L. King, Spectrochim. Acta, Part B, 2001, 56, 2449.
11 C. L. Lewis, G. P. Jackson, S. K. Doorn, V. Majidi and F. L. King, Spectrochim. Acta, Part B, 2001, 56, 487.

$12 \mathrm{~W}$. Hang, C. Baker, B. W. Smith, J. D. Winefordner and W. W. Harrison, J. Anal. At. Spectrom., 1997, 12, 143.

13 W. W. Harrison and W. Hang, J. Anal. At. Spectrom., 1996, 11, 835.

14 C. L. Lewis, M. A. Moser, D. E. Dale, Jr, W. Hang, D. C. Hassel, F. L. King and V. Majidi, Anal. Chem., 2003, 75, 1983.

15 L. Li, J. T. Millay, J. P. Turner and F. L. King, J. Am. Soc. Mass Spectrom., 2003, 15, 87.

16 J. Robertson-Honecker, Na. Zhang, A. Pavkovich and F. L. King, J. Anal. At. Spectrom., 2008, 23, 1508.

17 N. Zhang and F. L. King, J. Anal. At. Spectrom., 2009, 24, 1489.

18 J. A. Klingler, P. J. Savickas and W. W. Harrison, J. Am. Chem. Soc, 1990, 1, 138.

19 W. Hang, W. O. Walden and W. W. Harrison, Anal. Chem., 1996, 68, 1148 .

20 A. Bengtson, C. Yang and W. W. Harrison, J. Anal. At. Spectrom., 2000, 9, 1279

21 Y. P. Raizer, Gas Discharge Physics, ed. J. E. Allen, Springer, Berlin, Heidelberg, New York, 1991.

22 C. Yang, K. Ingeneri and W. W. Harrison, J. Anal. At. Spectrom., 1999, 14, 693.

23 M. Kasik, C. Michellon and L. C. Pitchford, J. Anal. At. Spectrom., 2002, 17, 1398.

24 V. Efimova, A. Derzsi, A. Zlotorowicz, V. Hoffmann, Z. Donko and J. Eckert, Spectrochim. Acta, Part B, 2010, 65, 311.

25 V. Efimova, M. Voronov, V. Hoffmann and J. Eckert, Publ. Astron. Obs. Belgrade, 2008, 84, 369.

26 V. Hoffmann, V. Efimova, M. V. Voronov, P. Smid, E. B. M. Steers and J. Eckert, J. Phys.: Conf. Ser., 2008, 133, 012017.

27 T. Nelis, M. Aeberhard, M. Hohl, L. Rohr and J. Michler, J. Anal. At. Spectrom., 2006, 21, 1.

28 X. Yan, K. Ingeneri, W. Hang and W. W. Harrison, J. Anal. At. Spectrom., 2001, 16, 819.

29 M. R. Winchester and K. R. Marcus, Anal. Chem., 1992, 64, 2067.

30 F. L. King and C. Pan, Anal. Chem., 1993, 63, 735.

31 A. Bogaerts and R. Gijbels, J. Anal. At. Spectrom., 2000, 15, 895.

32 M. Voronov and A. Ganeev, Spectrochim. Acta, Part B, 2009, 64, 416.

33 L. Wilken, V. Hoffmann, H.-J. Uhlemann, H. Siegel and K. Wetzig, J. Anal. At. Spectrom., 2003, 18, 646.

34 C. L. Lewis, L. Li, J. T. Millay, S. Downey, J. Warrick and F. L. King, J. Anal. At. Spectrom., 2003, 18, 527.

35 G. Gamez, A. Bogaerts and G. M. Hieftje, J. Anal. At. Spectrom., 2006, 21, 350.

36 V. Hoffmann, H.-J. Uhlemann, F. Präßler, K. Wetzig and D. Birus, Fresenius' J. Anal. Chem., 1996, 355, 826.

37 L. Wilken, V. Hoffmann and K. Wetzig, Spectrochim. Acta, Part B, 2007, 62, 1085.

38 W. W. Harrison and W. Hang, Fresenius' J. Anal. Chem., 1996, 355, 803.

39 A. Bogaerts, R. Gijbels and W. Goedheer, Spectrochim. Acta, Part B, 1999, 54, 1335.

40 Ph. Belenguer and J. P. Boeuf, Phys. Rev. A: At., Mol., Opt. Phys, 1990, 41, 4447.

41 A. Bogaerts and R. Gijbels, J. Tech. Phys., 2000, 41, 183.

42 F. Präßler, V. Hoffmann, J. Schumann and K. Wetzig, J. Anal. At. Spectrom., 1995, 9, 677.

43 K. A. Marshall, T. J. Casper, K. R. Brushwyler and J. C. Mitchell, J. Anal. At. Spectrom., 2003, 18, 637.

44 C. Yang, E. Oxley and W. W. Harrison, J. Anal. At. Spectrom., 2001, 16, 1131 .

45 A. Bogaerts, R. Gijbels and G. P. Jackson, J. Anal. At. Spectrom., 2003, 18, 533.

46 R. Payling, in Glow Discharge Optical Emission Spectrometry, ed. R. Payling, D. Jones and A. Bengtson, JOHN WILEY \& SONS, Chichester, New York, Weinheim, Brisbane, Singapore, Toronto, 1997, pp. 483-496.

47 P. W. J. M. Boumans, Anal. Chem., 1972, 44, 1219.

48 M. Voronov, V. Hoffmann, W. Buscher, C. Engelhard, S. J. Ray and G. M. Hieftje, J. Anal. At. Spectrom., 2010, in press. 